University of Nebraska - Lincoln

DigitalCommons@University of Nebraska - Lincoln

Faculty Publications, Department of Psychology

Psychology, Department of

2-4-2004

\title{
Hedonic capacity, cigarette craving, and diminished positive mood
}

Jessica Werth Cook

University of Illinois at Chicago, jwcook@ctri.wisc.edu

Bonnie Spring

University of Illinois at Chicago

Dennis E. McChargue

University of Nebraska-Lincoln, dmcchargue2@unl.edu

Donald Hedecker

University of Illinois at Chicago

Follow this and additional works at: https://digitalcommons.unl.edu/psychfacpub

Part of the Psychiatry and Psychology Commons

Werth Cook, Jessica; Spring, Bonnie; McChargue, Dennis E.; and Hedecker, Donald, "Hedonic capacity, cigarette craving, and diminished positive mood" (2004). Faculty Publications, Department of Psychology. 287.

https://digitalcommons.unl.edu/psychfacpub/287

This Article is brought to you for free and open access by the Psychology, Department of at DigitalCommons@University of Nebraska - Lincoln. It has been accepted for inclusion in Faculty Publications, Department of Psychology by an authorized administrator of DigitalCommons@University of Nebraska - Lincoln. 


\title{
Hedonic capacity, cigarette craving, and diminished positive mood
}

\author{
Jessica Werth Cook, Bonnie Spring, Dennis McChargue, Donald Hedeker
}

[Received 9 August 2002; accepted 11 March 2003]

Cigarette craving has been linked to elevated negative and positive moods, but a connection to deficient positive affect has not been studied. We tested whether a low hedonic capacity predicts a heightened urge to smoke after acute nicotine deprivation, and whether such an effect is mediated by decreased positive mood or increased negative mood. A total of 35 smokers characterized for individual differences in hedonic capacity were deprived of nicotine for $\mathbf{4 8} \mathrm{hr}$. Using mixed-effects regression modeling, we found that lower hedonic capacity predicted greater increases in craving $24 \mathrm{hr}$ after nicotine withdrawal, $t(29)=-2.33, p=.03$. The effect of hedonic capacity on increased 24-hr postquit craving to smoke was fully mediated by decreased positive affect. Findings suggest that in early nicotine withdrawal, smokers with diminished capacity to experience pleasure have heightened susceptibility to cigarette cravings that arises because of decreased positive mood rather than increased negative mood.

\section{Introduction}

Negative affect has long been associated with smoking behaviors. Findings suggest that susceptibility to negative mood states promotes nicotine dependence (Lumley, Downey, Stettner, Wehmer, \& Pomerleau, 1994) and increases vulnerability to smoking relapse (Hall et al., 1996; Rausch, Nichinson, Lamke, \& Matloff, 1990). Empirical research on affective disturbance comorbid with nicotine dependence has focused on identifying personality variables (e.g., depression-proneness, neuroticism) that predispose people toward dysphoric mood states (Gilbert, 1996). That research approach has been based on the plausible inference that smokers who are prone to negative affect learn habitually to self-administer nicotine via processes involving negative reinforcement. The presumption has been that because nicotine's pharmacological properties alleviate the negative moods to which they are prone, affectively vulnerable smokers undergo frequent operant conditioning trials

Jessica Werth Cook, M.A., and Donald Hedeker, Ph.D., University of Illinois at Chicago; Dennis McChargue, Ph.D., and Dennis McChargue, Ph.D., University of Illinois at Chicago and Edward Hines Jr. VA Hospital, Chicago, IL.

Correspondence: Bonnie Spring, Ph.D., Psychology Department (M/C 285), University of Illinois at Chicago, 1007 W. Harrison Street, Chicago, IL 60607, USA. Tel.: + 1 (312)-413-2628; Fax: + 1 (312)-3552155; E-mail: bspring@uic.edu during which they experience that smoking dispels negative mood (Carmody, 1992; Hall, Munoz, Reus, \& Sees, 1993).

What has been largely neglected is the prospect that personality variables associated with diminished positive affect, as opposed to elevated negative affect, might contribute to smoking behavior. That neglect is surprising in light of the evidence that deficiencies in positive mood represent the hallmark of depression (Clark \& Watson, 1991; Coyne, 1994), which is associated with nicotine dependence (Breslau, Kilbey, \& Andreski, 1993; Hall et al., 1996). Some evidence suggests that nicotine may directly enhance positive affect (Perkins, Grobe, \& Fonte, 1997; Warburton \& Mancuso, 1998) or indirectly increase the reward value of pleasurable situations (HustonLyons \& Kornetsky, 1992). Those findings raise the possibility that smokers with low hedonic capacity might self-administer nicotine to enhance their ability to experience positive affect.

Positive affect is defined as a pleasant and energized mood state that reflects feelings of elation, excitement, enthusiasm, and peppiness (Watson \& Tellegen, 1985). The dearth of research examining diminished positive mood as a potential determinant of smoking may reflect a theoretical view that positive and negative affect are redundant constructs. To the extent that the two are simply opposite poles along a single affective 
dimension (Russell \& Carroll, 1999), positive mood should reflect little more than an absence of negative mood. Empirically, however, positive and negative affects are correlated though distinct (Watson, Clark, \& Tellegen, 1988), link to different neural underpinnings (Davidson, 1992), and have different psychological correlates (Watson et al., 1988).

We hypothesized that individual differences in hedonic capacity prior to quitting smoking would predict the degree to which cigarette cravings increase during acute nicotine deprivation, and that this association would be explained by a decrease in postquit positive moods and possibly by an increase in postquit negative moods. Hedonic capacity is defined as the ability to experience positive affect in response to situations that are typically rewarding (Meehl, 1975 , 1987). Lowered positive affective response to typically rewarding stimuli is thought to reflect diminished function in brain dopamine systems that mediate reward (Phillips, 1984). At the lowest end of the hedonic spectrum are those with anhedonia, which is characterized by severe deficits in the capacity to experience positive affect. Anhedonia has been identified as a symptom of both schizophrenia (Chapman, Chapman, \& Raulin, 1976; Meehl, 1975) and depression (Berenbaum \& Oltmanns, 1992). Anhedonia differs from alexithymia (generalized blunting of both positive and negative emotions) in that the dearth of emotional experience is confined to positive affect (Fiorito \& Simons, 1994).

To date, research on hedonic capacity has been characterized largely by a personal deficit approach: Focusing on that sector of the population that exhibits prominent, psychopathologically significant levels of anhedonia. What has been overlooked is the observation that hedonic capacity is a personality trait that has a normal distribution in the population (Fawcett, Clark, Scheftner, \& Gibbons, 1983; Meehl, 1975, 1987). At the upper end of this spectrum are those who respond easily and fully to life's pleasures. High hedonic capacity may even be an adaptive personality attribute that helps people cope with hardships (Meehl, 1975, 1987). For example, positive affect has been shown to offset physiological byproducts of negative affect (Fredrickson \& Levenson, 1998), perhaps preventing a decline into depression (Folkman \& Moskowitz, 2000; Fredrickson \& Levenson). Conversely, reduced hedonic capacity might theoretically be a vulnerability factor that predisposes toward the development of depression (Fawcett et al., 1983; Loas, 1996; Meehl, 1975, 1987), via a dearth of positive emotions with which to buffer stress.

We posit that by self-administering nicotine, a dopamine releaser, during exposure to ordinary life pleasures, smokers with lowered hedonic capacity may be able to pharmacologically stimulate neural reward systems that environmental rewards readily trigger for those with a higher hedonic capacity. To the extent that smokers with a low hedonic capacity have few other effective ways to induce positive mood states, they should crave cigarettes strongly when nicotine is withdrawn, and their heightened craving should be mediated by losses in positive mood. Additionally, to the extent that smokers with low hedonic capacity lack positive emotional reserves that could help them buffer against dysphoria, negative mood arising from nicotine withdrawal also might contribute to increased craving.

The present study tested the following hypotheses about hedonic capacity and cigarette smoking: (a) Lower hedonic capacity will be predictive of greater increases in cigarette craving during 24- and 48-hr nicotine deprivation, (b) the influence of low hedonic capacity on increased craving after quitting smoking will be mediated by decreased positive mood, and (c) the influence of low hedonic capacity on heightened cigarette craving also may be mediated by increased negative moods.

\section{Method}

\section{Participants}

Participants ( $n=35 ; 51 \%$ female) were smokers who ranged in age between 18 and 65 years $(M=36.80$, $S D=11.74)$ and had smoked for an average of 17.50 years $(S D=11.03)$ (Table 1$)$. We recruited participants via fliers distributed to businesses and supermarkets throughout a large midwestern city. Flyers advertised a paid research study for which smokers were required to abstain from nicotine for $48 \mathrm{hr}$. Entry criteria required candidates to have smoked at least 10 cigarettes a day for the past year. Those who currently used nicotine replacement, actively abused alcohol or drugs, or received alcohol or drug abuse treatment

Table 1. Demographic and smoking characteristics $(n=35)$.

\begin{tabular}{lcrc}
\hline Variable & Mean & \multicolumn{1}{c}{$S D$} & Range \\
\hline Age (years) & 36.8 & 11.74 & $20-62$ \\
FTQ & 5.6 & 2.09 & $2-10$ \\
Number of years smoked & 17.50 & 11.03 & $1-40$ \\
Hedonic capacity & 123.00 & 11.53 & $98-144$ \\
Positive affect & & & \\
$\quad$ Baseline & 13.03 & 7.71 & $0-32$ \\
24-hr postquit & 9.25 & 6.59 & $0-32$ \\
48-hr postquit & 10.56 & 6.85 & $0-32$ \\
Negative affect & & & \\
Baseline & 8.10 & 8.04 & $0-36$ \\
24-hr postquit & 20.09 & 19.67 & $.67-91.33$ \\
48-hr postquit & 19.16 & 16.49 & $2-58.33$ \\
Craving & & & \\
Baseline & 28.63 & 6.47 & $14-39$ \\
24-hr postquit & 30.41 & 8.22 & $7-42$ \\
48-hr postquit & 26.44 & 9.07 & $6-42$ \\
\end{tabular}

$S D$, standard deviation; FTQ, Fagerström Tolerance Questionnaire (Fagerström, 1978). 
within the past year were excluded from participation on the basis of a telephone-screening interview. Those who had current axis I diagnoses other than nicotine dependence also were excluded via a diagnostic interview. A total of 40 candidates were excluded from study enrollment because of failure to meet entry criteria: $31(78 \%)$ because they smoked less than the required 10 daily cigarettes, $4(9.5 \%)$ because they met criteria for substance abuse or dependence, and 5 $(12.5 \%)$ because they met criteria for current major depressive disorder. An additional five eligible people chose not to enroll or were unable to be reached after screening. Participants gave informed consent after receiving a full explanation of study procedures. All 35 enrollees maintained abstinence for $48 \mathrm{hr}$, as indexed by expired-air carbon monoxide verification, and received a US\$75 honorarium for completion of the study.

\section{Measures}

Axis I disorders. The Structured Clinical Interview for $D S M$ - $I V$-Nonpatient version (SCID-NP; Spitzer, Williams, Gibbon, \& First, 1992) was administered by trained clinicians to assess for axis I disorders. The SCID-NP has moderate construct validity, as shown by its favorable comparison with other diagnostic assessment methods (Williams et al., 1992). The SCID-NP was used to operationalize entry criteria that screened out candidates who met criteria for substance abuse or dependence, posttraumatic stress disorder or other anxiety disorders, major depressive disorder, or dysthymia.

Smoking characteristics. The eight-item Fagerström Tolerance Questionnaire (FTQ; Fagerström, 1978) yields a potential score range from 0 to 11 ; higher scores indicate greater physical dependence on nicotine. Correlations between the FTQ and measures of nicotine intake support the construct validity of the scale (Fagerström \& Schneider, 1989). Participants also provided breath samples that were analyzed immediately for expired-air carbon monoxide via an ecolyzer (Model EC-50, Vitalograph Corporation). Criteria for continuous 48-hr nicotine abstinence required both self-report of zero cigarettes smoked and ecolyzer assessments showing carbon monoxide less than 8 parts per million after both 24 and $48 \mathrm{hr}$ of deprivation.

Hedonic capacity. The Fawcett-Clark Pleasure Scale (FCPS; Fawcett et al., 1983) measured hedonic capacity by asking how subjects usually react to 36 situations typically experienced as enjoyable. Using Likert scales $(1=$ no pleasure at all; $5=$ extreme and lasting pleasure), respondents rated how pleasurable they would find events such as embracing a loved one or witnessing their grown child's success. Scores on the FCPS can range from 36 to 180; lower scores indicate diminished hedonic capacity. Internal consistency for the FCPS is very high: Cronbach's alpha for the present study was .89. FCPS scores have been shown to remain stable over a 1-year time span, suggesting that the scale measures a stable, trait-like attribute (Clark, Fawcett, SalazarGrueso, \& Fawcett, 1984).

Craving. The Shiffman-Jarvik Smoking Withdrawal Questionnaire (SWQ; Shiffman \& Jarvik, 1976) was administered to assess the effects of smoking deprivation on changes in craving for a cigarette. Comprising six Likert scale items $(1=$ definitely; $7=$ definitely not), the craving subscale has a potential score range of 6 to 42 . Internal consistency for the present study was strong: Cronbach's alphas ranged from .82 to .92 for craving at baseline and 24- and 48-hr postquit.

Negative and positive affect. The Profile of Mood States (POMS; McNair, Lorr, \& Droppleman, 1971) is a self-report mood questionnaire consisting of 65 adjectives rated on Likert scales $(0=$ not at all; $4=$ extremely). The "right now" version was used to characterize participants' mood at the time of testing. The POMS yields six factor-analytically derived scaled scores that all possess evidence of construct and predictive validity (McNair et al., 1971). Negative affect, encompassing feelings of distress, hostility, nervousness, scorn, and gloominess (Watson \& Tellegen, 1985), was measured by the POMS dysphoria score, derived by summing the subscale scores for tension, depression, and anger. Negative affect scores could range between 0 and 144. Cronbach's alphas for POMS dysphoria in the present study ranged between .86 and .97 across assessment times, indicating strong internal consistency. Positive affect, manifested by feelings of activation, elation, enthusiasm, and peppiness (Watson \& Tellegen, 1985), was measured by the POMS vigor subscale, which assesses moods such as happiness, jubilation, and peppiness. Vigor subscale scores potentially range between 0 and 32. Internal consistency for vigor in the present study was excellent: Cronbach's alphas ranged between .92 and .96 across all measurement times.

\section{Procedure}

Screening assessment. Assenting candidates' demographic information, smoking history, and general health history were gathered via an initial telephone interview. During a subsequent laboratory visit, project staff administered the FTQ (Fagerström, 1978) to assess nicotine dependence, and the SCID-NP 
diagnostic interview (Spitzer et al., 1992) to identify and exclude candidates with comorbid psychopathology other than nicotine dependence.

Baseline assessment while smoking. Candidates who passed the screening evaluations completed baseline assessments including the FCPS to assess hedonic capacity, the POMS to measure positive and negative affect, and the SWQ to measure craving. Participants smoked a cigarette $30 \mathrm{~min}$ before completing the questionnaires to minimize effects of nicotine withdrawal on their responses. They filled out POMS questionnaires in their natural environments, completing them in the morning and at noon. Then they came to the laboratory in the early evening and filled out the evening POMS, before completing the FCPS and SWQ.

Nicotine deprivation assessment. One week after baseline assessment, participants quit smoking for a 48-hr period of bioverified nicotine deprivation. Over the course of the 2-day abstinence period, they completed the POMS mood questionnaire (McNair et al., 1971) six times: In the morning, noon, and early evening of both days of abstinence (Figure 1). Participants visited the laboratory at 5:00 P.M. each day (24 and $48 \mathrm{hr}$ after discontinuing smoking). While there, they completed the evening POMS, followed by the SWQ to measure cigarette cravings. To verify abstinence from smoking, we measured expired-air carbon monoxide during each laboratory visit.

\section{Results}

\section{Analytic plan}

The analytic plan tested the predictions that hedonic capacity influenced the change in cigarette craving during the initial $48 \mathrm{hr}$ of nicotine deprivation and that changes in positive affect or negative affect mediated the association between hedonic capacity and postquit change in craving. Longitudinal analysis of craving, positive, and negative affect (baseline and 24- and 48-hr postquit) was conducted using mixedeffects regression modeling, implemented via SAS PROC MIXED. In these analyses, we characterized the change in each dependent variable in terms of two time-related contrasts: $24-\mathrm{hr}$ contrast (24-hr postquit to baseline difference) and 48-hr contrast (48-hr postquit to baseline difference). Thus, interactions with these time-related contrasts (e.g., hedonic capacity by $24-\mathrm{hr}$ contrast and hedonic capacity by $48-\mathrm{hr}$ contrast) indicate the influence of hedonic capacity on the change from baseline to 24-hr postquit, and from baseline to 48 -hr postquit, respectively. Additionally, gender was treated as a covariate in all analyses. The variance-covariance matrix of the repeated measures was modeled using random subject intercepts and time trends. As recommended, this variance covariance structure for the longitudinal data was selected as being most parsimonious only after comparison with several other potential structures (Verbeke \& Molenberghs, 2000).

Mixed-effects regression modeling does not place restrictions on the number of observations per individual, so that participants with missing data at a particular assessment time were not excluded from analyses. Instead, model parameters were estimated

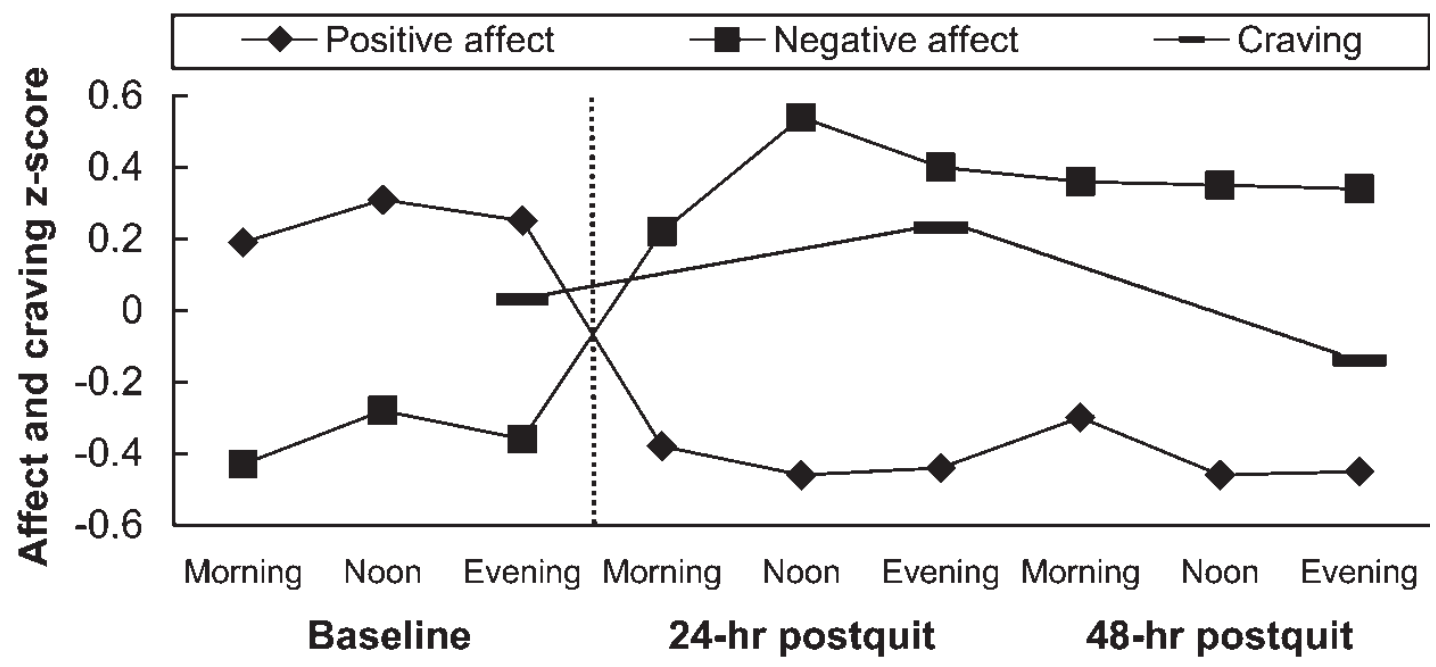

Figure 1. Levels of positive affect, negative affect, and craving during baseline (while smoking) and during $48 \mathrm{hr}$ of nicotine deprivation. Positive and negative affects were measured three times during baseline and six times during nicotine deprivation. Craving was measured once during baseline and twice during nicotine deprivation. Mean levels of positive affect, negative affect, and craving were converted to $z$-scores so that they could be graphed on the same scale. 
Table 2. Intercorrelations among predictor and criterion variables $(n=35)$.

\begin{tabular}{|c|c|c|c|c|c|c|c|}
\hline & 1 & 2 & 3 & 4 & 5 & 6 & 7 \\
\hline 1. Hedonic capacity & - & & & & & & \\
\hline 2. Change in craving ( $24 \mathrm{hr}$ ) & $-.47^{\star \star}$ & - & & & & & \\
\hline 3. Change in craving ( $48 \mathrm{hr}$ ) & -.13 & $.64^{\star \star}$ & - & & & & \\
\hline 4. Change in positive affect ( $24 \mathrm{hr}$ ) & $.40^{\star}$ & $-.54^{\star *}$ & -.10 & - & & & \\
\hline 5. Change in positive affect ( $48 \mathrm{hr}$ ) & .33 & $-.35^{\star}$ & -.14 & $.86^{\star *}$ & - & & \\
\hline 6. Change in negative affect ( $24 \mathrm{hr}$ ) & -.20 & .14 & .13 & -.28 & -.30 & - & \\
\hline 7. Change in negative affect ( $48 \mathrm{hr}$ ) & -.29 & .28 & .18 & $-.37^{\star}$ & $-.46^{\star}$ & $.77^{\star *}$ & - \\
\hline
\end{tabular}

${ }^{*} p<.05,{ }^{* *} p<.01$, one-tailed.

using all available data. The model assumes that the data present for a given subject reasonably reflect that subject's deviation from the usual fixed-effects regression part of the model (i.e., the regressors multiplied by their coefficients). Missing data were examined after affect data were aggregated into baseline, 24-hr, and $48-\mathrm{hr}$ postquit assessments. Three measures of hedonic capacity were missing. For craving, three baseline, one 24-hr, and one 48-hr measurement were missing. Four baseline, four 24-hr, and three 48-hr POMS were missing. The total $N$ effective for all analyses was 35 subjects times 3 assessment times, or 105 possible observations minus the data points missing for the particular analysis.

\section{Preliminary analyses}

Mood and craving stability during baseline and $48 \mathrm{hr}$ of nicotine deprivation. To determine the legitimacy of constructing composite positive and negative affect scores at baseline and the two postquit intervals, we evaluated the stability of mood scores across each assessment interval via repeated-measures analysis of variance (ANOVA). Analyses detected no significant changes as a function of repeated testing for positive or negative affect scores within either the baseline or the nicotine deprivation intervals (all $F$ values ranged from .79 to $1.55, p=n s$ ) (Figure 1). Given the stability of affect scores, repeated measures were averaged to create baseline, 24-hr postquit, and 48-hr postquit positive and negative affect variables.

Correlational analyses. Correlations were computed to identify appropriate covariates. Of the potential covariates (FTQ, age, amount smoked, gender), only gender correlated significantly with any dependent variable (changes in negative affect; $r=.38, p=.01$ ). Gender was, therefore, covaried out of the mixedeffects regression modeling. Correlational analyses also tested for overlap among the primary study variables. Results showed that hedonic capacity was not significantly correlated with positive affect $(r=.17, p=.20)$ or negative affect $(r=.09, p=.31)$ when all three were measured while participants were still smoking, consistent with the premise that hedonic capacity represents a different construct from negative and positive affects. Positive and negative affects were inversely correlated at baseline $(r=-.29, p=.07)$ and during 24 and $48 \mathrm{hr}$ of nicotine deprivation $(r=-.53, p=.01$, and $r=-.49$, $p=.01$, respectively), although not to the extent that they appeared to reflect the same construct. Finally, correlations were examined between hedonic capacity and postquit changes in positive affect, negative affect, and craving at both 24 and $48 \mathrm{hr}$. As shown in Table 2, hedonic capacity was significantly correlated with changes in craving and positive affect during the first $24 \mathrm{hr}$ of nicotine deprivation. Greater hedonic capacity was associated with decreased craving and increased positive affect during the first $24 \mathrm{hr}$ of nicotine deprivation. Reduced hedonic capacity, in contrast, was associated with increased craving and decreased postquit positive affect at 24-hr postquit (Figures 2 and 3).

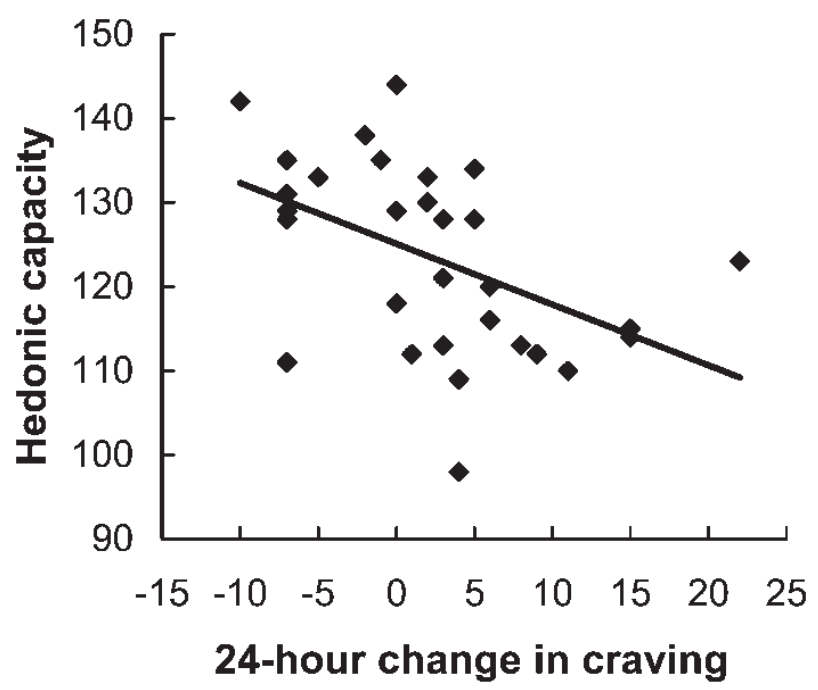

Figure 2. Scatterplot depicting the relationship between hedonic capacity (higher scores = greater pleasure capacity) and postquit changes in craving (craving after $24 \mathrm{hr}$ of nicotine deprivation minus baseline craving while smoking). 


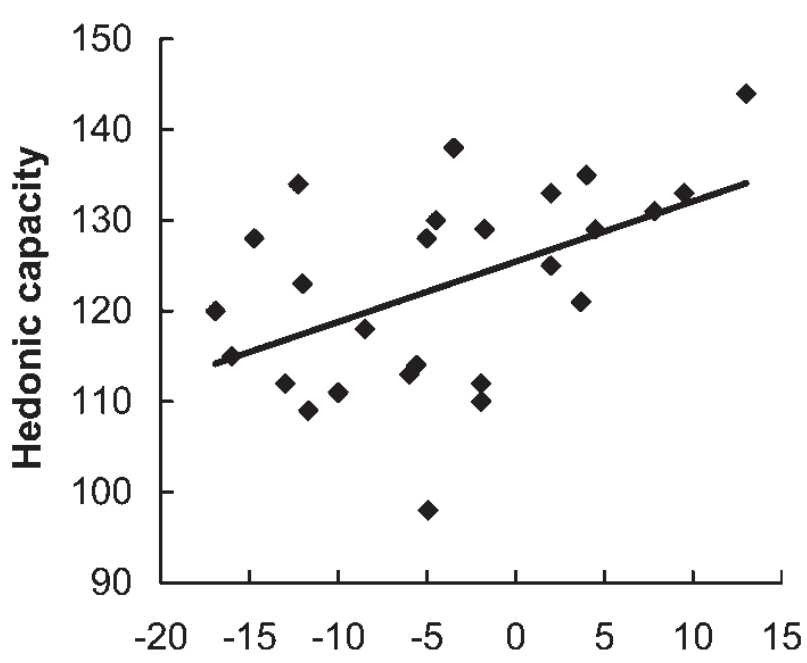

24-hour change in positive affect

Figure 3. Scatterplot depicting the relationship between hedonic capacity and 24-hr postquit changes in positive affect.

\section{Hedonic capacity and postquit changes in craving}

Longitudinal analysis showed a significant interaction between hedonic capacity and the 24-hr contrast, $t(26)=-2.33, p=.03$, indicating that hedonic capacity predicted change in craving after $24 \mathrm{hr}$ of nicotine deprivation. As shown by a nonsignificant interaction between hedonic capacity and the 48-hr contrast, $t(26)=-.81, p=.42$ (Table 3 ), however, hedonic capacity no longer retained its influence on change in craving 48-hr postquit. Thus, as illustrated in Figure 2, lower hedonic capacity predicted an increase in craving 24-hr postquit, and higher hedonic capacity predicted a smaller increase or even a decrease in craving over the same time period.

\section{Hedonic capacity and changes in craving: Mediation} by changes in positive affect and not negative affect

Next we examined the hypotheses that decreased positive affect and increased negative affect would

Table 3. Step 1 mediation: Predictors of changes in craving 24- and 48-hr postquit, determined by mixed-effects regression modeling.

\begin{tabular}{lcrc}
\hline Variable & $\begin{array}{c}\text { Regression } \\
\text { coefficient }\end{array}$ & \multicolumn{1}{c}{$S E$} & $t$ \\
\hline Covariates & & & \\
$\quad$ Gender & 4.32 & 2.03 & $2.13^{*}$ \\
$\quad$ Hedonic capacity & -.09 & .11 & -.84 \\
$\begin{array}{l}\text { Time effects } \\
\quad \text { 24-hr contrast }\end{array}$ & 30.16 & 12.51 & $2.41^{*}$ \\
$\quad$ 48-hr contrast & 12.04 & 16.35 & .74 \\
$\begin{array}{l}\text { Interactions } \\
\text { Hedonic capacity } \times 24-\mathrm{hr} \\
\quad \text { contrast }\end{array}$ & -.24 & .10 & $-2.33^{*}$ \\
$\quad$ Hedonic capacity $\times 48-\mathrm{hr}$ & -.11 & .13 & -.81 \\
$\quad$ contrast & & & \\
\hline
\end{tabular}

${ }^{*} p<.05$. mediate the relationship between lower hedonic capacity and greater increase in craving during the first $24 \mathrm{hr}$ of nicotine deprivation. Negative affect did not meet criteria for mediation, since 24-hr postquit changes in negative affect were not significantly correlated with either hedonic capacity or 24-hr postquit change in craving (Table 2) (Baron \& Kenny, 1986). Thus, only change in positive affect $24 \mathrm{hr}$ after quitting was examined as a mediator of the relationship between hedonic capacity and change in craving 24-hr postquit. Based on criteria set forth by Baron and Kenny, mixed-effects regression showed that, after controlling for gender, the interaction between hedonic capacity and the 24-hr contrast significantly predicted change in positive affect after $24 \mathrm{hr}$ of nicotine deprivation, $t(20)=2.28, p=.03$ (Table 4). As illustrated in Figure 3, lower hedonic capacity predicted a greater postquit decline in positive affect, whereas higher hedonic capacity predicted a lesser decline or even an increase in positive affect 24-hr postquit. Next, when the positive affect by 24-hr contrast was placed as a covariate into the full model, the hedonic capacity by $24-\mathrm{hr}$ contrast no longer predicted significant 24-hr change in craving, $t(16)=-1.18, p=.25$. The positive affect by 24-hr contrast, however, predicted a significant increase in 24-hr postquit craving, $t(16)=-2.29$, $p=.04$ (Table 5). Further, the regression coefficient for the effect of hedonic capacity on change in 24-hr postquit craving was divided in half when change in positive affect was added into the model, $B=-.24$ to $B=-.12$ (Tables 3 and 5).

Partial correlational analysis offered a simpler statistical demonstration that change in positive affect after the first 24-hr postquit mediates the relationship between hedonic capacity and changes in craving during the same time period. Table 2 shows the significant, $r=-.47, p=.01$, Pearson correlation between hedonic capacity and 24-hr postquit changes in craving. Controlling for changes in positive affect 24-hr postquit, however, caused the relationship

Table 4. Step 2 mediation: Predictors of changes in positive affect 24- and 48-hr postquit, determined by mixedeffects regression modeling.

\begin{tabular}{lrrr}
\hline & $\begin{array}{c}\text { Regression } \\
\text { coefficient }\end{array}$ & SE & $t$ \\
\hline Cariable & & & \\
$\quad$ Genariates & -4.26 & 2.15 & -1.98 \\
$\quad$ Hedonic capacity & .15 & .12 & 1.19 \\
$\begin{array}{l}\text { Time effects } \\
\quad 24-h r \text { contrast }\end{array}$ & -29.77 & 12.47 & $-2.39^{*}$ \\
$\quad \begin{array}{l}\text { 48-hr contrast } \\
\text { Interactions } \\
\quad \text { Hedonic capacity } \times 24-\mathrm{hr} \\
\quad \text { contrast }\end{array}$ & -24.35 & 15.62 & -1.56 \\
$\quad$ Hedonic capacity $\times$ 48-hr & .23 & .10 & $2.28^{*}$ \\
$\quad$ contrast & .18 & .13 & 1.48 \\
\hline
\end{tabular}

${ }^{*} p<.05$. 
Table 5. Step 3 mediation: Predictors of changes in craving 24- and 48-hr postquit, determined by mixed-effects regression modeling.

\begin{tabular}{lrrr}
\hline Variable & $\begin{array}{c}\text { Regression } \\
\text { coefficient }\end{array}$ & \multicolumn{1}{c}{$S E$} & \multicolumn{1}{c}{$t$} \\
\hline Covariates & & & \\
$\quad$ Gender & 2.98 & 2.06 & 1.44 \\
$\quad$ Hedonic capacity & -.18 & .12 & -1.45 \\
$\quad$ Positive affect & -.15 & .14 & -1.08 \\
Time effects & & & \\
$\quad \begin{array}{l}\text { 24-hr contrast } \\
\text { 48-hr contrast }\end{array}$ & 21.70 & 13.17 & 1.65 \\
Interactions & 5.03 & 18.75 & .27 \\
$\quad$ Hedonic capacity $\times 24-\mathrm{hr}$ & -.12 & .11 & -1.18 \\
$\quad$ contrast & & .15 & -.14 \\
$\quad$ Hedonic capacity $\times 48-\mathrm{hr}$ & -.02 & .16 & $-2.29^{*}$ \\
$\quad$ contrast & & & \\
$\quad \begin{array}{l}\text { Positive affect } \times 24-\mathrm{hr} \\
\text { contrast }\end{array}$ & -.37 & .22 & -.91 \\
$\begin{array}{l}\text { Positive affect } \times 48-\mathrm{hr} \\
\text { contrast }\end{array}$ & -.91 & & \\
\hline
\end{tabular}

${ }^{*} p<.05$.

between hedonic capacity and changes in craving to become nonsignificant, $r=-.27, p=.10$. These results are consistent in indicating that decreased positive affect 24-hr postquit mediates the relationship between hedonic capacity and increased craving over the same time period.

\section{Discussion}

Lower hedonic capacity was predictive of greater increases in craving after $24 \mathrm{hr}$ of nicotine deprivation. Decreases in positive moods at 24-hr postquit, but not increases in negative moods, mediated the influence of diminished hedonic capacity on increased craving during early nicotine deprivation. Overall, these results indicate that smokers with a lower pleasure capacity experienced a heightened rise in cigarette craving during the initial $24 \mathrm{hr}$ of nicotine deprivation, which was explained by a disproportionate decline in their positive affect.

Results suggest that the heightened loss in positive affect that relatively anhedonic smokers experience during early nicotine deprivation generates their increased urge to smoke. Also, decreased positive affect was predictive of heightened postquit craving, regardless of level of pleasure capacity. That observation is of particular importance in highlighting the widespread relevance of diminished positive affect as a withdrawal-related emotional change that augments the urge to smoke. The findings suggest a need to broaden existing theory about how affective sequelae of nicotine withdrawal influence the risk of smoking relapse. Our data show that changes in positive emotion, in general, may be as important as changes in negative emotion in triggering urges to smoke, and for some individuals (i.e., those with low hedonic capacity) diminished positive emotion is more important.

It is interesting to consider these observations in view of prevailing theories that link affect with urges to smoke. In their two-affect model of drug motivation, Baker, Morse, and Sherman (1986) differentiated drug use urges that arise in tandem with negative affect from those that arise in conjunction with the pursuit of positive affect. That model attributed a postquit rise in urges to smoke to the increment in negative mood that usually accompanies withdrawal from nicotine. Baker et al. (1986) also addressed a link between cigarette craving and fluctuation in positive moods during continued smoking but not in the context of nicotine withdrawal. The phenomenon of diminished positive affect during nicotine deprivation has not been addressed, nor has the prospect that diminished positive mood states rather than elevated negative ones could stimulate craving during nicotine deprivation.

Except for Baker et al.'s (1986) dual-affect theory, alternative models of substance use motivation do not explicitly discuss positive mood or hedonic capacity. Rather, they emphasize the appetitive pursuit of rewards, which may or may not be associated with positive mood states and hedonic capacity (Robinson \& Berridge, 2000; Watkins, Koob, \& Markou, 2000). Our findings may be most compatible with a developmental model of drug addiction presented by Koob and colleagues. They presented evidence that chronic nicotine exposure produces an elevation of hedonic set point (Koob \& LeMoal, 1997) that persists through smoking abstinence (Epping-Jordan, Watkins, Koob, \& Markou, 1998). An elevated reward threshold is thought to arise from neuroadaptations reflecting lowered dopamine neurotransmission (Koob \& Lemaol). A natural consequence might be a diminished hedonic capacity, such that ordinary pleasures lose some of their potency as dopamine releasers and, hence, some of their rewarding impact. Anhedonic smokers may learn to jumpstart sluggish positive affective responses by priming their reward pathways with a direct dopaminereleasing agent such as nicotine. We speculate that, even for the average smoker, lowering of positive affect observed in early nicotine deprivation prompts a rise in urges to smoke because of the anticipation that nicotine self-administration can release dopamine and enhance positive mood states. The mediational pathway linking loss of positive affect to heightened urge to smoke may be especially strong for smokers with diminished hedonic capacity because they acquire considerable experience in self-administering nicotine to overcome positive mood deficits.

The present study had various limitations. First, the sample was somewhat small and comprised healthy smokers. Generalizability to populations with greater 
physical and psychological comorbidities cannot be assumed. Second, the observed changes in craving and affect cannot conclusively be attributed to nicotine deprivation, because other sensory and psychological changes also accompany smoking discontinuation. Third, craving was measured only in the evening and, as such, may have been influenced by time-of-day effects. Fourth, craving remained elevated only for the 24-hr assessment and diminished by $48 \mathrm{hr}$. These findings are not surprising given the transient nature of craving and the differing contexts that surrounded the 24- and 48-hr assessments. Participants' longing for a cigarette may have been blunted at the 48-hr assessment by their awareness that they could soon smoke, unlike at the 24-hr assessment when they knew that 24 more hrs of nicotine deprivation lay ahead. Finally, in view of the correlational nature of the data, no inferences about causality can be made.

We were surprised to observe that change in negative affect did not predict change in postquit craving, particularly since overall level of negative affect often correlates with overall craving level and relapse risk (Hall et al., 1993). In that regard, note that our aim in the present study was to determine what changes in psychological processes produce the heightened cigarette cravings that make the first few days of nicotine withdrawal so uncomfortable. Piasecki et al. (2000) found that a rise in postcessation negative affect and craving was strongly associated with an increased likelihood of relapse to smoking. Given that change in affect contributes uniquely to understanding withdrawal processes associated with relapse (Piasecki et al.), we examined whether intensification of cigarette cravings in the early postquit period is explicable on the basis of sudden undesirable affective changes. Our interest in understanding relationships among acute changes in affect and craving contrasts with the more typical approach of examining relationships among absolute levels of these constructs. Our findings indicated that acute exacerbation of craving in early nicotine withdrawal is better explained by deterioration in positive affect than by worsening of negative affect. That is a different question than whether people who have higher negative affect generally and during nicotine withdrawal also tend to have higher craving. Thus, our results in no way diminish the likely importance of typical levels of negative affect in influencing the longer-term development of nicotine dependence.

The present findings demonstrate that declines in positive affect are an important aspect of the phenomenology of nicotine withdrawal, particularly because they also are associated with heightened cigarette cravings. Results indicate that smokers with an elevated hedonic capacity undergo a lesser decline or even an increase in positive affect after quitting smoking, which mediates their diminished urges to smoke. Such findings suggest that elevated hedonic capacity may be an adaptive characteristic that buffers the stress of nicotine deprivation. Conversely, relatively more anhedonic smokers are prone to experience larger losses in positive affect after quitting, which explains their elevated cravings to smoke. Results, if borne out, have implications for tailoring smoking cessation treatments. If enhancement of positive moods proves to be a primary "hook" that binds smokers with low hedonic capacity to their cigarettes, their greatest challenge in quitting smoking may be to find new behavioral options to activate an underresponsive brain reward system. Even normally hedonic smokers might benefit from engaging during the quit period in pleasurable activities that prevent a loss of positive mood with an accompanying rise in urges to smoke.

\section{Acknowledgments}

This research was supported by a VA Merit Review award and HL52577 to Bonnie Spring, as well as by K08DA00467-01 and a VA Merit Review Entry Program to Dennis McChargue and DA14144-01 to Jessica Werth. The authors thank Regina Pingitore, Dianne Gunnarsdottir, Brian Hitsman, and Michele Pergadia for their help with this project.

\section{References}

Baker, T. B., Morse, E., \& Sherman, J. E. (1986). The motivation to use drugs: A psychobiological analysis of urges. In C. Rivers (Ed.), The Nebraska symposium on motivation: Alcohol use and abuse (pp. 257-323). Lincoln: University of Nebraska Press.

Baron, R. M., \& Kenny, D. A. (1986). The moderator-mediator variable distinction in social psychological research: Conceptual, strategic, and statistical considerations. Journal of Personality and Social Psychology, 51(6), 1173-1182.

Berenbaum, H., \& Oltmanns, T. F. (1992). Emotional experience and expression in schizophrenia and depression. Journal of Abnormal Psychology, 101, 37-44.

Breslau, N., Kilbey, M. M., \& Andreski, P. (1993). Nicotine dependence and major depression: New evidence from a prospective investigation. Archives of General Psychiatry, 50, 31-35.

Carmody, T. P. (1992). Affect regulation, nicotine addiction, and smoking cessation. Journal of Psychoactive Drugs, 24(2), 111-122.

Chapman, L., Chapman, J., \& Raulin, M. (1976). Scales for physical and social anhedonia. Journal of Abnormal Psychology, 85, 374-382.

Clark, D. C., Fawcett, J., Salazar-Grueso, E., \& Fawcett, E. (1984). Seven-month clinical outcome of anhedonic and normally hedonic depressed inpatients. The American Journal of Psychiatry, 141, 1216-1220.

Clark, L. A., \& Watson, D. (1991). Tripartite model of anxiety and depression: Psychometric evidence and taxonomic implications. Journal of Abnormal Psychology, 100(3), 316-336.

Coyne, J. C. (1994). Self-reported distress: Analog or ersatz depression? Psychological Bulletin, 116(1), 29-45.

Davidson, R. J. (1992). Anterior asymmetry and the nature of emotion. Brain and Cognition, 20, 125-151.

Epping-Jordan, M. P., Watkins, S. S., Koob, G. F., \& Markou, A. (1998). Dramatic decreases in brain reward function during nicotine withdrawal. Nature, 393, 76-79.

Fagerström, K. O. (1978). Measuring degree of physical dependence to tobacco smoking with reference to individualization of treatment. Addictive Behaviors, 3, 235-241.

Fagerström, K. O., \& Schneider, N. G. (1989). Measuring nicotine dependence: A review of the Fagerström Tolerance Questionnaire. Journal of Behavioral Medicine, 12(2), 159-182.

Fawcett, J., Clark, D. C., Scheftner, W. A., \& Gibbons, R. D. (1983). Assessing anhedonia in psychiatric patients. Archives of General Psychiatry, 40, 79-84. 
Fiorito, E. R., \& Simons, R. F. (1994). Emotional imagery and physical anhedonia. Psychophysiology, 31, 513-521.

Folkman, S., \& Moskowitz, J. T. (2000). Positive affect and the other side of coping. The American Psychologist, 55, 647-654.

Fredrickson, B. L., \& Levenson, R. W. (1998). Positive emotions speed recovery from cardiovascular sequelae of negative emotions. Cognition and Emotion, 12, 191-220.

Gilbert, D. G. (1996). Depression, smoking, and nicotine: Toward a bioinformational situation by trait model. Drug Development, 38, 267-277.

Hall, S. M., Munoz, F., Reus, V. I., \& Sees, K. L. (1993). Nicotine, negative affect, and depression. Journal of Consulting and Clinical Psychology, 61(5), 761-767.

Hall, S. M., Munoz, R. F., Reus, V. I., Sees, K. L., Duncan, C., Humfleet, G. L., \& Hartz, D. T. (1996). Mood management and nicotine gum in smoking treatment. Journal of Consulting and Clinical Psychology, 62, 141-146.

Huston-Lyons, D., \& Kornetsky, C. (1992). Effects of nicotine on the threshold for rewarding brain stimulation. Pharmacology, Biochemistry, and Behavior, 41, 755-759.

Koob, G. F., \& LeMoal, M. (1997). Drug abuse: Hedonic homeostatic dysregulation. Science, 278, 52-58.

Loas, G. (1996). Vulnerability to depression: A model centered on anhedonia. Journal of Affective Disorders, 41, 39-53.

Lumley, M. A., Downey, K., Stettner, L., Wehmer, F., \& Pomerleau, O. F. (1994). Alexithymia and negative affect: Relationship to cigarette smoking, nicotine dependence, and smoking cessation. Psychotherapy \& Psychosomatics, 61(3-4), 156-162.

McNair, D. M., Lorr, M., \& Droppleman, L. F. (1971). Profile of Mood States manual. San Diego, CA: Educational and Industrial Testing Service.

Meehl, P. E. (1975). Hedonic capacity: Some conjectures. Bulletin of the Menninger Clinic, 39, 295-307.

Meehl, P. E. (1987). Hedonic capacity ten years later: Some clarifications. In C. C. Clark \& J. Fawcett (Eds.), Anhedonia and affect deficit states. New York: PMA Publishing.

Perkins, K. A., Grobe, J., \& Fonte, C. (1997). Influence of acute smoking exposure on the subsequent reinforcing value of smoking. Experimental and Clinical Psychopharmacology, 5(3), 277-285.
Phillips, A. G. (1984). Brain reward circuitry: A case for separate systems. Brain Reward Bulletin, 12, 195-201.

Piasecki, T. M., Niaura, R., Shadel, W. G., Abrams, D., Goldstein, M., Fiore, M. C., \& Baker, T. B. (2000). Smoking withdrawal dynamics in unaided quitters. Journal of Abnormal Psychology, 109(1), 74-86.

Rausch, J. L., Nichinson, B., Lamke, C., \& Matloff, J. (1990). Influence of negative affect on smoking cessation outcome: A pilot study. British Journal of Addictions, 85, 929-933.

Robinson, T. E., \& Berridge, K. C. (2000). The psychology and neurobiology of addiction: An incentive-sensitization view. Addiction, 95(Suppl. 2), S91-S117.

Russell, J. A., \& Carroll, J. M. (1999). On the bipolarity of positive and negative affect. Psychological Bulletin, 125(1), 3-30.

Shiffman, S., \& Jarvik, M. E. (1976). Smoking withdrawal symptoms in two weeks of abstinence. Psychopharmacology, 50, 35-39.

Spitzer, R. L., Williams, J. B., Gibbon, M., \& First, M. B. (1992). The Structured Clinical Interview for DSM-III-R (SCID). History, rationale, and description. Archives of General Psychiatry, 49, 624-629.

Verbeke, G., Molenberghs, G., \& (Eds.) (2000). Linear mixed models for longitudinal data. New York: Springer.

Warburton, D. M., \& Mancuso, G. (1998). Evaluation of the information processing and mood effects of a transdermal nicotine patch. Psychopharmacology, 135, 305-310.

Watkins, S. S., Koob, G. F., \& Markou, A. (2000). Neural mechanisms underlying nicotine addiction: Acute positive reinforcement and withdrawal. Nicotine \& Tobacco Research, 2, 19-37.

Watson, D., Clark, L. A., \& Tellegen, A. (1988). Development and validation of brief measures of positive and negative affect: The PANAS scales. Journal of Personality and Social Psychology, 54, 1063-1070.

Watson, D., \& Tellegen, A. (1985). Toward a consensual structure of mood. Psychological Bulletin, 98(2), 219-235.

Williams, J. B. W., Gibbon, M., First, M. B., Spitzer, R. L., Davies, M., Borus, J., Howes, M. J., Kane, J., Pope, H. G., Rounsaville, B., \& Wittchen, H. U. (1992). The Structured Clinical Interview for DSM-III-R (SCID). II. Multisite test-retest reliability. Archives of General Psychiatry, 49, 630-636. 